\title{
Effect of Polymerization Statistics on the Electronic Properties of Copolymers for Organic Photovoltaics
}

Gabriele Boschetto, ${ }^{\dagger}$ Hong-Tao Xue, ${ }^{\dagger} \uparrow$ Jacek Dziedzic, ${ }^{\dagger, \S}$ Michal Krompiec, ${ }^{\ddagger}$ and Chris-Kriton Skylaris*, $\dagger$

$\dagger$ School of Chemistry, University of Southampton, Highfield, Southampton SO17 1BJ, United Kingdom

$\ddagger$ Merck Chemicals Ltd., Chilworth Technical Centre, University Parkway, Southampton SO16 7QD, United Kingdom

IState Key Laboratory of Advanced Processing and Recycling of Non-Ferrous Metals, Department of Materials Science and Engineering, Lanzhou University of Technology, Lanzhou 730050, People's Republic of China

$\S$ Faculty of Applied Physics and Mathematics, Gdansk University of Technology, Gdansk, Poland

E-mail: c.skylaris@soton.ac.uk

Phone: +44 (0)2380 599381 


\begin{abstract}
Statistical block copolymers, composed of donor (D) and acceptor (A) blocks, are a novel type of material for organic photovoltaics (OPVs) devices. In particular a new series of polymers based on PBTZT-stat-BDTT-8, recently developed by Merck, offers high solubility in different solvents, and a high power conversion efficiency (PCE) in different device architectures. Although it is known that the electronic properties of these materials may be significantly affected by attaching different functional groups on different blocks, it is not fully clear how important the influence of the polymer composition (i.e. the $\mathrm{D} / \mathrm{A}$ block ratio) is, even if previous studies suggest that this might also have an effect. Therefore, the effect of the polymer composition in terms of HOMO, LUMO energies and band gap was explored by studying a number of long chain oligomers with more than 1000 atoms each, and with different D/A ratios. This study, that is novel both conceptually and methodologically, was made possible by using the linear-scaling reformulation of DFT implemented in the ONETEP code. Our results showed that changing the composition has a significant effect on the electronic structure of statistical copolymers, making this an alternative and suitable strategy to obtain materials with desired properties. Also, a systematic analysis of the effect of a range of different substituents placed in the D and A blocks of PBTZT-stat-BDTT-8 was performed in order to investigate how this class of materials responds to functionalization. We found that it is not possible to know a priori using chemical intuition what kind of influence different types of functional groups may have on these systems, and therefore computational modelling is essential.
\end{abstract}

\title{
Introduction
}

The world energy consumption is constantly growing and therefore there is a pressing need to find new and alternative sources of energy in both industry and academia. Technologies based on renewable and clean energies do exist, and among these, photovoltaics show great promise. Organic photovoltaics (OPV) based on $\pi$-conjugated polymers in particular have drawn 
attention in recent years because of their low manufacturing costs, solution processability and flexibility, not to mention the fact that these devices are lightweight, and also efficient in diffuse light and indoors. ${ }^{1}$ Moreover, despite their relatively low performance, they show a very fast energy payback time (EPBT): while for silicon-based technologies the EPBT is in the order of years, for OPV it has been estimated to be in the order of days. ${ }^{2,3}$

The active layer of an OPV module, in the state-of-the-art bulk heterojunction (BHJ) architecture,${ }^{4}$ consists of a blend of electron-donor and electron-acceptor materials, the first being typically a conjugated polymer and the latter being in the majority of cases a soluble fullerene derivative ([6,6]-phenyl- $\mathrm{C}_{61}$-butyric acid methyl ester, also known as $\mathrm{PC}_{61} \mathrm{BM}$ or simply PCBM). This kind of interpenetrating network allows the exciton splitting and the subsequent generation of an electron and a hole in the form of free charge carriers. Since the photoabsorption happens in the polymer bulk, the photocurrent conversion efficiency (PCE) largely depends on the number of photons that are absorbed by the polymer. One of the most used materials in BHJ devices was poly(3-hexylthiophene) (P3HT), a regioregular homopolymer that led to modules showing a PCE of $6 \% \cdot{ }^{5,6}$ One of the problems of P3HT, however, lied in its relatively large band $\operatorname{gap}^{7}(\sim 2 \mathrm{eV})$ that doesn't allow the absorption of a large number of photons, since its absorption spectrum overlaps with just a small part of the solar emission spectrum; it has in fact been estimated that P3HT is able to collect in the best case scenario only $\sim 20 \%$ of the solar photons. ${ }^{8}$ Different strategies were then proposed with the aim of lowering the band gap of these materials, in oder to harvest photons at longer wavelengths and therefore absorb a wider portion of the solar radiation that reaches the Earth: ${ }^{9}$ one of these is the synthesis of donor-acceptor (D-A, or push-pull) copolymers, formed by alternating electron-rich and electron-deficient blocks. This leads to an intramolecular charge transfer (ICT) process between the D and A blocks, ${ }^{10}$ and to materials having in general a smaller and tunable band gap with respect to homopolymers like P3HT mentioned above; this effect is due to the orbital mixing between the two different units. The band gap (and consequently HOMO and LUMO energies) can be carefully tuned 
by combining different acceptor and donor units and/or by changing the functional groups attached to those blocks. In addition, recent studies suggest that modifying the composition (i.e. the donor-acceptor ratio) of the material can lead to different HOMO and LUMO energies. ${ }^{11}$ This finding can potentially offer a novel strategy for the design of polymers with desired electronic properties. In general it is desirable to reduce the band gap while maintaining at the same time a low HOMO level so that a high open-circuit voltage $\left(V_{\mathrm{OC}}\right)$ value, and in turn a high efficiency, can be obtained, as proposed by Scharber and coworkers. ${ }^{12}$ It has in fact been demonstrated empirically that for BHJ solar cells with PCBM as the acceptor species, an approximate linear relation exists between the HOMO energy of the conjugated polymer and the $V_{\mathrm{OC}}$ of the device:

$$
V_{\mathrm{OC}}=(1 / e)\left(\left|E_{H O M O}^{\text {Donor }}\right|-\left|E_{L U M O}^{P C B M}\right|\right)-0.3 \mathrm{~V}
$$

where $e$ is the elementary charge, the LUMO energy of PCBM is taken as a constant, and $0.3 \mathrm{~V}$ is an empirical value. Moreover ideally, in order to have a good hole mobility throughout a copolymer chain, valence bands (in other words HOMO energies) of different blocks should be similar.

One of the top D-A polymers in terms of efficiency, solubility and solution processability is part of the PBTZT-stat-BDTT-8 series, developed by Merck and employed in the solar tree installation at the German pavillion in Milan EXPO 2015. ${ }^{13}$ This royal-blue polymer, with a band gap of $1.7 \mathrm{eV}$, shows a 9\% PCE in lab scale and 4.5\% PCE in a fully roll-to-roll (R2R) module, the latter being the highest reported performance of flexible R2R semitransparent modules. PBTZT-stat-BDTT-8 is a novel class of OPV materials, consisting of statistical block copolymers composed of substituted benzo[1,2-b:4,5-b']dithiophene (BDT), thiophene (T), and 2,1,3-benzothiadiazole (BTZ). The fact that this material is a statistical copolymer means that blocks may have different lengths, different electronic properties, and may not be present with a 1:1 ratio in the chain: in fact it has been observed that these materials 
behave differently with respect to regioregular analogues. ${ }^{14}$ In addition, due to their reduced regularity, they show a higher solubility in nonhalogenated solvents, and this is of great importance from an industrial perspective, since widely used solvents such as chlorobenzene (CB) or ortho-dichlorobenzene (o-DCB) are not suitable for mass production. ${ }^{15}$

The main focus of this paper is the effect of composition, i.e. the effect of different block ratios in the copolymer chain, in terms of HOMO, LUMO energies and band gap, by studying a set of $\sim 1000$-atom oligomers, each one with a different $\mathrm{D} / \mathrm{A}$ block ratio. This study, which is both conceptually and methodologically novel, was enabled by using a linear-scaling DFT approach that allowed the investigation of the electronic properties of these systems at a far larger scale than possible before. We have also studied the effect of a range of different substituents both in the donor and acceptor units of some analogues of PBTZT-stat-BDTT-8, to investigate how and in what measure the electronic properties of this class of statistical copolymers can be affected by replacing the side chains in the D and A blocks with electron-donating and electron-withdrawing functional groups.

This study has been carried out using density functional theory calculations. The methods that have been used are described in Section 2, including the set-up details of the simulations, while in Section 3 results are presented, together with their interpretation in the context of OPVs. Finally conclusions are discussed in Section 4.

\section{Methods}

\section{The ONETEP program}

All simulations were performed in the density functional theory (DFT) framework of electronic structure theory using the ONETEP (Order-N Electronic Total Energy Package) code for linear-scaling DFT. ${ }^{16,17}$ Linear-scaling reformulations of DFT have been known, but suffer from reduced accuracy (i.e. only work with small basis sets); the peculiarity of ONETEP is that it combines linear-scaling behaviour with large basis set accuracy, allowing efficient 
calculations also on systems composed of thousands of atoms. To achieve linear-scaling behaviour, DFT has been reformulated in terms of the one-particle density matrix. In terms of Kohn-Sham orbitals the density matrix can be expressed as follows

$$
\rho\left(\mathbf{r}, \mathbf{r}^{\prime}\right)=\sum_{n=0}^{\infty} f_{n} \psi_{n}(\mathbf{r}) \psi_{n}^{*}\left(\mathbf{r}^{\prime}\right)
$$

where $f_{n}$ is the occupancy and $\psi_{n}(\mathbf{r})$ are the Kohn-Sham orbitals. In ONETEP the density matrix assumes the form

$$
\rho\left(\mathbf{r}, \mathbf{r}^{\prime}\right)=\sum_{\alpha} \sum_{\beta} \phi_{\alpha}(\mathbf{r}) K^{\alpha \beta} \phi_{\beta}^{*}\left(\mathbf{r}^{\prime}\right)
$$

where $\phi_{\alpha}(\mathbf{r})$ are local orbitals called nonorthogonal generalized Wannier functions (NGWFs ${ }^{18}$ and $K$ is known as the density kernel. Making the density kernel sparse by truncation and enforcing strict localization of the NGWFs onto atomic regions leads to linear-scaling behaviour. To achieve plane-wave accuracy, both the density kernel and the NGWFs, that are confined into spherical regions centered on each one of the atoms, are optimized during

the calculation. The NGWFs are expanded in a basis set of periodic sinc (psinc) functions ${ }^{19}$ equivalent to a plane-wave basis, allowing to easily control the basis set quality with just one parameter, equivalent to the kinetic energy cutoff in conventional DFT codes that make use of plane waves.

\section{Simulation Details}

Three limiting cases of structures were considered: $x=0, y=1$ and $x=1, x=0$, where $x$ and $y$ are the number of units for each block, BDT-T and BTZ-T respectively, as shown in Figure 1; in other words each single block alone was studied, as well as structures with alternating electron-rich and electron-deficient units. The effect of different substituents was then explored, in particular: $\mathrm{R}_{1}, \mathrm{R}_{2}=\mathrm{H}, \mathrm{F}, \mathrm{OCH}_{3}, \mathrm{CH}_{3}, \mathrm{CN}$; in a few cases the $\mathrm{OCH}_{3}$ and $\mathrm{CH}_{3}$ 


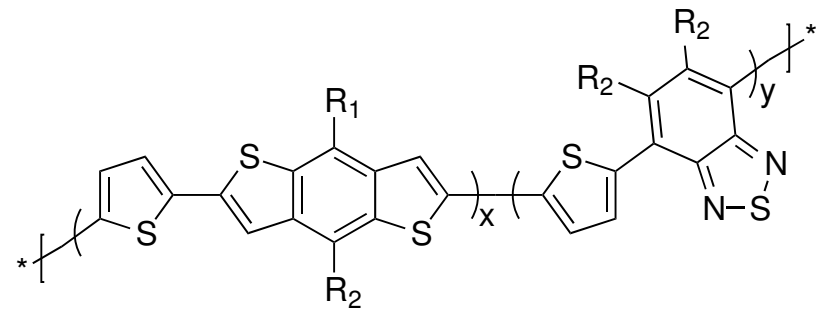

Figure 1: Structure of PBTZT-stat-BDTT-8, with $x$ being the BDT-T block (donor, D) and $y$ the BTZ-T block (acceptor, A).

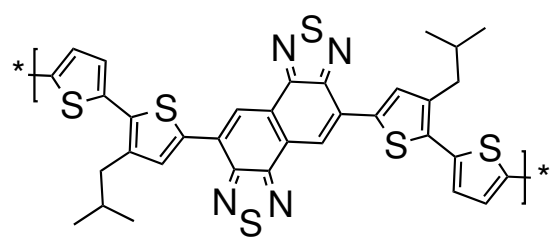

PNTz4T-2OD

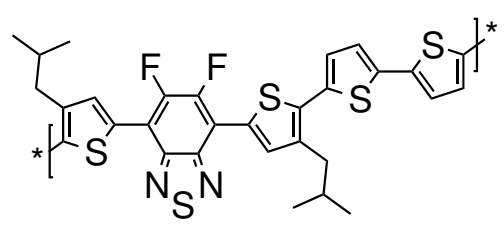

PffBT4T-2OD

Figure 2: Structures of PNTz4T-2OD and PffBT4T-2OD, two examples of high-performing polymers.

groups were also replaced with the bulkier $\mathrm{OC}\left(\mathrm{CH}_{3}\right)_{3}$ and $\mathrm{C}\left(\mathrm{CH}_{3}\right)_{3}$ groups respectively, in order to test the effect of steric hindrance on the electronic structure of these materials. The effect of fluorination on thiophene rings was also considered, as well as the replacement of benzothiadiazole with naphtho[1,2-c:5,6-c']bis[1,2,5]thiadiazole (NTZ). As comparative examples, two high-performing polymers were also taken into consideration: PffBT4T-2OD ${ }^{20}$ and PNT4T-2OD ${ }^{21}$ (see Figure 2). The electronic structures of both repeat units and polymers were studied, with a specific focus on the HOMO and LUMO levels, as well as the band gap $\left(E_{g}\right)$; the structures of polymers were obtained using periodic boundary conditions (PBC) as implemented in ONETEP. When needed, in order to converge band gaps, more than a single unit was used to build the repeating cells. Note that for structures with $x=0$ and $y=0$ (i.e. BTZ-T, A only, and BDT-T, D only) a single unit consisted of two alternated benzothiadiazole and thiophene moieties, and two alternated benzodithiophene and thiophene units, respectively. It is worth mentioning that besides PBC, other methods to predict band gaps of conjugated polymers have been reported in literature, such as the extrapolation of the linear (or quadratic) curve of the band gap against $1 / n$, where $n$ is the 
number of monomer units. ${ }^{22} \mathrm{~A}$ comparative study between those methods for a subset of structures studied in this paper is available in the Supporting Information.

Finally, to overcome the limitation of being able to treat only perfectly alternating copolymers and to allow the study of polymer chains with different block ratios, five oligomers, each one composed of a total sum of $40 \mathrm{D}$ and A blocks, consisting of 1000 atoms were built using the Materials Studio ${ }^{23}$ software by setting different values of reactivity ratios for each block; their geometries were then optimized and their electronic structures were analyzed. The reactivity ratios used in this work refer to the Mayo-Lewis equation ${ }^{24}$ (known also as copolymer equation) and they describe whether a monomer A is more likely to react with itself rather than with a monomer B during a copolymerization reaction; if the reactivity ratios are known, then it is possible to predict the final composition of the resulting copolymer. Note that every oligomer presents the same functional groups in $R_{1}$ and $R_{2}$ positions, that is $\mathrm{CH}_{3}$ and $\mathrm{OCH}_{3}$ respectively. In order to avoid the effect of chain twisting and to make all results comparable, the starting guess structure for each oligomer before geometry optimization was a flat and linear conformation. Table 1 shows the reactivity ratios of the $\mathrm{D}$ and $\mathrm{A}$ units used for each oligomer, together with the number of $\mathrm{D}$ and $\mathrm{A}$ blocks that compose each oligomer, and the total number of atoms.

Table 1: Reactivity ratios used in this work for BDT-T (D unit) and BTZ-T (A unit) for each studied oligomer, together with the number of $D$ and $A$ blocks present in each chain, and the total number of atoms.

\begin{tabular}{ccccc}
\hline$r_{D}$ & $r_{A}$ & no. D blocks & no. A blocks & no. atoms \\
\hline 10 & 0.1 & 38 & 2 & 1156 \\
5 & 0.2 & 36 & 4 & 1150 \\
0.2 & 5 & 9 & 31 & 1069 \\
0.1 & 10 & 4 & 36 & 1054 \\
1 & 1 & 18 & 22 & 1096 \\
\hline
\end{tabular}

All the ground-state energy calculations were done in vacuum using the Perdew-BurkeErnzerhof exchange-correlation functional $(\mathrm{PBE})^{25}$ with the projector augmented wave method (PAW). It is well known that despite giving a good description of the properties of a chemical 
system, pure functionals, such PBE, fail to accurately describe the $E_{g}$ of semiconductors: the width of the gap is underestimated. ${ }^{26,27} \mathrm{~A}$ better approximation of the $E_{g}$ can be obtained with hybrid exchange-correlation functionals such as B3LYP. ${ }^{28,29}$ Since the available ONETEP version allows for periodic calculations using non-hybrid functionals only, ${ }^{30}$ the hybrid functional B3LYP was also used together with norm-conserving pseudopotentials only when running simulations on repeat units. After calibration of the method, optimal values for the kinetic energy cutoff and the NGWFs radii were found to be respectively $800 \mathrm{eV}$ and 9.0 bohr, and they were used for all the simulations.

\section{Results and Discussion}

\section{Electronic levels and band gaps}

For each studied structure (see Figure 1 and Figure 2), detailed results of the energy levels (HOMO, LUMO and $E_{g}$ ) are shown in Table 2 both for repeat units and polymers, and in Figure 3 and Figure 4 for repeat units and polymers respectively. Structures from 1 to 12 all consist of alternating D-A blocks (note that in each structure the A unit corresponds to BTZ-T, with the only exception of 12 in which the BTZ-T moiety has been substituted with NTZ-T), structures from 13 to 16 consist of only A blocks (BTZ-T), while the ones from 17 to 21 are composed of just D blocks (BDT-T). Lastly, structures 22 and 23 are PNT4T-2OD and PffBT4T-2OD respectively, two high-performing polymers studied in literature, as shown in Figure 2.

Results show that on average, both for repeat units and polymers, structures 13-16 composed of electron-deficient BTZ-T (A unit) present smaller gaps with respect to structures 17-21 composed of electron-rich BDT-T (D unit); structures 1-12, obtained by combining the previously discussed A and D units, present intermediate values as expected. In addition it can be seen that electron-withdrawing groups like $\mathrm{F}$ and $\mathrm{CN}$ tend to stabilize, and therefore shift to lower values, both HOMO and LUMO levels in both A and D units, while 
the opposite effect is observed for electron-donating groups like $\mathrm{CH}_{3}$ and $\mathrm{OCH}_{3}$.
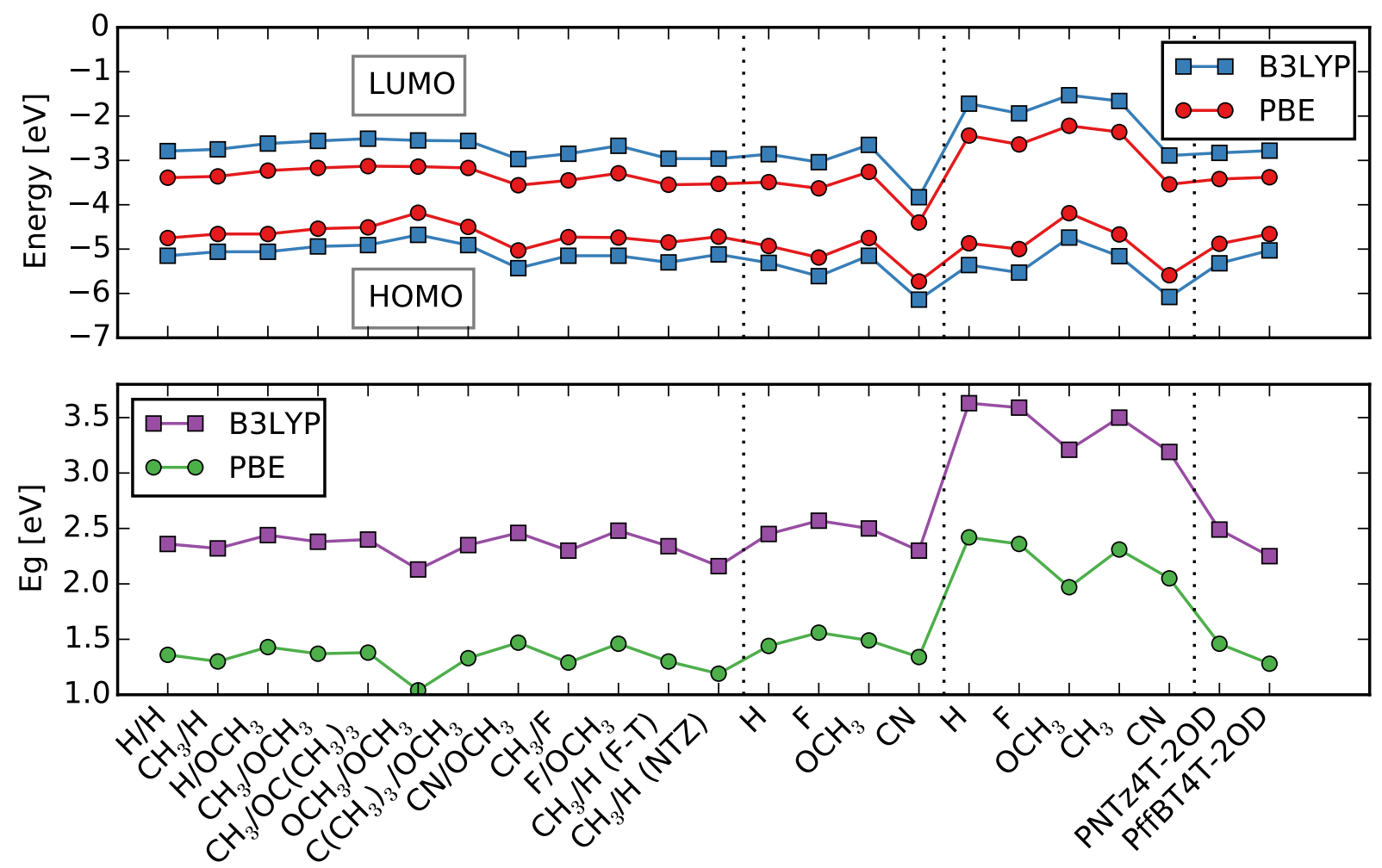

Repeat Unit

Figure 3: Graphical comparison of HOMO, LUMO and $E_{g}$ of repeat units obtained with ONETEP using PBE and B3LYP functionals.

Focusing on the energy gaps of repeat units, it is quite remarkable that not only the values obtained using the B3LYP functional follow the same trend of the ones obtained with PBE, but also that their absolute difference is rather constant, and close to $1 \mathrm{eV}$, as shown in Figure 3. The same behaviour can be observed for HOMO and LUMO energies: the effect of B3LYP functional is to shift HOMO (LUMO) levels towards lower (higher) values, but keeping the overall trend unaltered. In particular the shift in HOMO energies ranges between $0.4-0.5 \mathrm{eV}$, while for LUMO energies the range is between $0.6-0.7 \mathrm{eV}$.

Considering now the polymers, band gaps of structures from 1 to 12 range between $0.77 \mathrm{eV}$ for structure $6\left(\mathrm{R}_{1}=\mathrm{R}_{2}=\mathrm{OCH}_{3}\right)$ to $1.05 \mathrm{eV}$ for structure $10\left(\mathrm{R}_{1}=\mathrm{F}, \mathrm{R}_{2}=\mathrm{OCH}_{3}\right)$. Even though chemistry considerations would suggest that the strategy to reduce the band 
Table 2: HOMO, LUMO and $E_{g}$ obtained with ONETEP for all the repeat units and polymer structures studied in this paper. $^{a}$

\begin{tabular}{|c|c|c|c|c|c|c|c|c|c|c|}
\hline \multirow[b]{3}{*}{$\#$} & \multirow[b]{3}{*}{$\mathrm{R}_{1} / \mathrm{R}_{2}$} & \multicolumn{6}{|c|}{ Repeat units } & \multicolumn{3}{|c|}{ Polymers } \\
\hline & & \multicolumn{3}{|c|}{ energies $(\mathrm{eV})-\mathrm{PBE}$} & \multicolumn{3}{|c|}{ energies $(e V)$ - B3LYP } & \multicolumn{3}{|c|}{ energies $(\mathrm{eV})$ - PBE } \\
\hline & & $\mathrm{HOMO}$ & LUMO & $E_{g}$ & HOMO & LUMO & $E_{g}$ & $\mathrm{HOMO}$ & LUMO & $E_{g}$ \\
\hline 1 & $\mathrm{H} / \mathrm{H}$ & -4.75 & -3.39 & 1.36 & -5.15 & -2.79 & 2.36 & -4.13 & -3.22 & 0.91 \\
\hline 2 & $\mathrm{CH}_{3} / \mathrm{H}$ & -4.66 & -3.36 & 1.30 & -5.06 & -2.75 & 2.32 & -4.04 & -3.17 & 0.87 \\
\hline 3 & $\mathrm{H} / \mathrm{OCH}_{3}$ & -4.66 & -3.23 & 1.43 & -5.06 & -2.62 & 2.44 & -4.06 & -3.05 & 1.01 \\
\hline 4 & $\mathrm{CH}_{3} / \mathrm{OCH}_{3}$ & -4.54 & -3.17 & 1.37 & -4.94 & -2.56 & 2.38 & -4.20 & -3.25 & 0.95 \\
\hline 5 & $\mathrm{CH}_{3} / \mathrm{OC}\left(\mathrm{CH}_{3}\right)_{3}$ & -4.51 & -3.13 & 1.38 & -4.91 & -2.51 & 2.40 & -3.92 & -2.95 & 0.97 \\
\hline 6 & $\mathrm{OCH}_{3} / \mathrm{OCH}_{3}$ & -4.18 & -3.14 & 1.04 & -4.68 & -2.55 & 2.13 & -3.75 & -2.98 & 0.77 \\
\hline 7 & $\mathrm{C}\left(\mathrm{CH}_{3}\right)_{3} / \mathrm{OCH}_{3}$ & -4.50 & -3.17 & 1.33 & -4.91 & -2.56 & 2.35 & -3.94 & -2.98 & 0.96 \\
\hline 8 & $\mathrm{CN} / \mathrm{OCH}_{3}$ & -5.03 & -3.56 & 1.47 & -5.43 & -2.97 & 2.46 & -4.84 & -3.80 & 1.03 \\
\hline 9 & $\mathrm{CH}_{3} / \mathrm{F}$ & -4.73 & -3.45 & 1.29 & -5.15 & -2.85 & 2.30 & -4.48 & -3.58 & 0.90 \\
\hline 10 & $\mathrm{~F} / \mathrm{OCH}_{3}$ & -4.74 & -3.29 & 1.46 & -5.15 & -2.67 & 2.48 & -4.51 & -3.46 & 1.05 \\
\hline 11 & $\mathrm{CH}_{3} / \mathrm{H}(\mathrm{F}-\mathrm{T})$ & -4.85 & -3.55 & 1.30 & -5.30 & -2.96 & 2.34 & -4.66 & -3.67 & 0.99 \\
\hline 12 & $\mathrm{CH}_{3} / \mathrm{H}(\mathrm{NTZ})$ & -4.72 & -3.53 & 1.19 & -5.12 & -2.96 & 2.16 & -4.46 & -3.61 & 0.85 \\
\hline 13 & $\mathrm{H}$ & -4.93 & -3.49 & 1.44 & -5.31 & -2.86 & 2.45 & -4.43 & -3.68 & 0.75 \\
\hline 14 & $\mathrm{~F}$ & -5.19 & -3.63 & 1.56 & -5.61 & -3.04 & 2.57 & -4.74 & -3.88 & 0.86 \\
\hline 15 & $\mathrm{OCH}_{3}$ & -4.75 & -3.26 & 1.49 & -5.15 & -2.65 & 2.50 & -4.24 & -3.37 & 0.87 \\
\hline 16 & $\mathrm{CN}$ & -5.73 & -4.40 & 1.34 & -6.14 & -3.83 & 2.30 & -5.57 & -4.61 & 0.96 \\
\hline 17 & $\mathrm{H}$ & -4.87 & -2.44 & 2.42 & -5.36 & -1.72 & 3.63 & -4.48 & -3.12 & 1.36 \\
\hline 18 & $\mathrm{~F}$ & -5.00 & -2.64 & 2.36 & -5.53 & -1.94 & 3.59 & -4.75 & -3.39 & 1.36 \\
\hline 19 & $\mathrm{OCH}_{3}$ & -4.19 & -2.22 & 1.97 & -4.74 & -1.53 & 3.21 & -3.96 & -2.81 & 1.15 \\
\hline 20 & $\mathrm{CH}_{3}$ & -4.67 & -2.36 & 2.31 & -5.16 & -1.66 & 3.50 & -4.34 & -3.01 & 1.33 \\
\hline 21 & $\mathrm{CN}$ & -5.59 & -3.54 & 2.05 & -6.08 & -2.89 & 3.19 & -5.29 & -4.13 & 1.16 \\
\hline 22 & PNTz4T-2OD & -4.88 & -3.42 & 1.46 & -5.32 & -2.83 & 2.49 & -4.26 & -3.49 & 0.77 \\
\hline 23 & PffBT4T-2OD & -4.66 & -3.38 & 1.28 & -5.03 & -2.78 & 2.25 & -4.29 & -3.46 & 0.83 \\
\hline
\end{tabular}

${ }^{a} \mathrm{R}_{1}$ and $\mathrm{R}_{2}$ are the functional groups in the $\mathrm{D}$ and $\mathrm{A}$ units respectively. Structures 11 and 12 have the same functional groups as structure 2, but the first one has been fluorinated on the $\mathrm{T}$ rings in position 3 and 4, while in the second one the BTZ moiety has been substituted with NTZ.

Note that structures from 13 to 16 (BTZ-T, A units only), and from 17 to 21 (BDT-T, D units only) present one functional group only. Structures 22 and 23 are not part of the PBTZT-stat-BDTT-8 series, and therefore only their names are shown. 

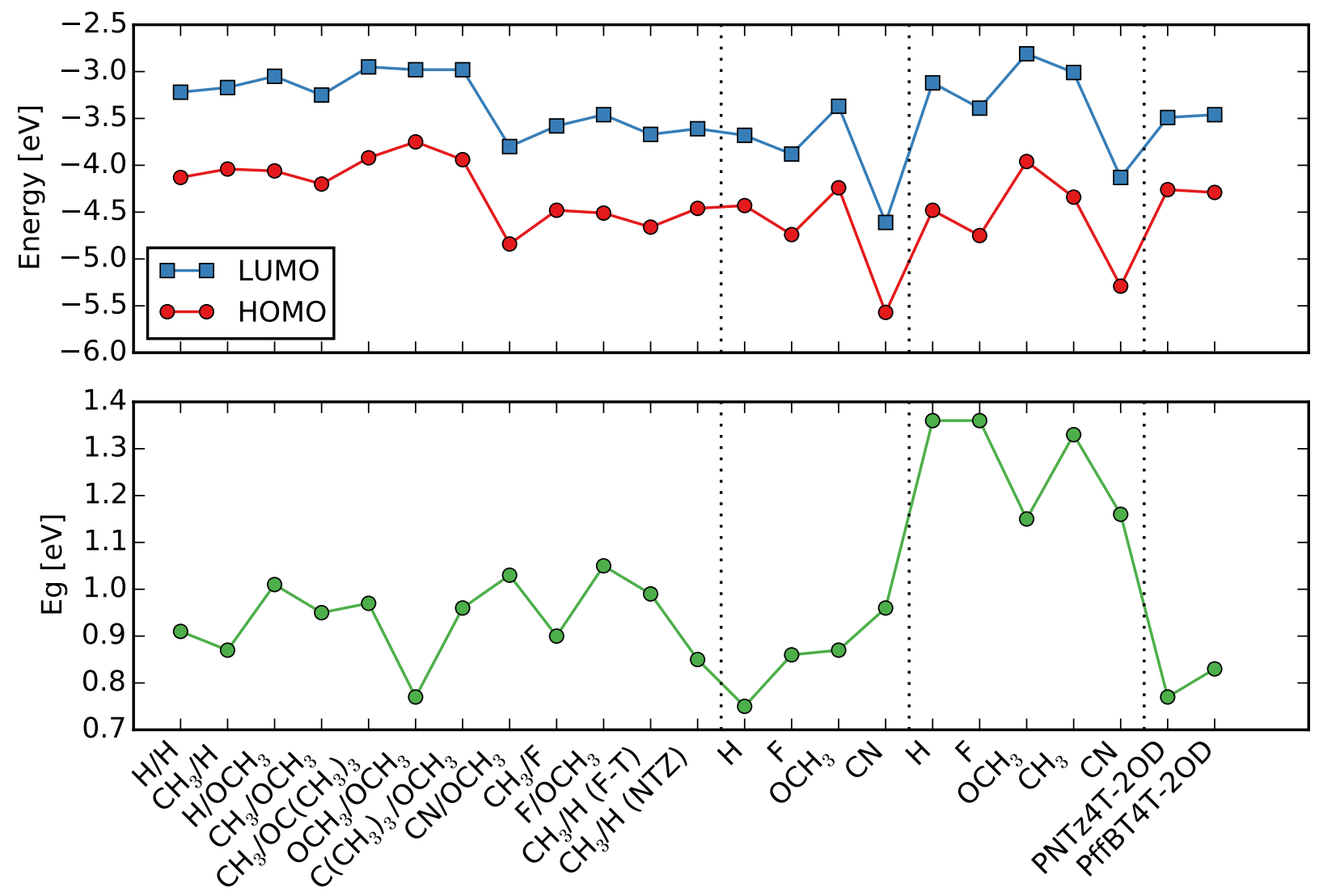

Polymer

Figure 4: Graphical comparison of HOMO, LUMO and $E_{g}$ of polymers obtained with ONETEP using the PBE functional with PBC. 
gap would be to increase the HOMO energy by attaching an electron-donating group on the D unit, and to reduce LUMO energy by attaching an electron-withdrawing group on the A unit, results show that this isn't necessarily true: structure 6 for example presents the lowest band gap in the series despite having electron-donating methoxy groups in both $\mathrm{D}$ and $\mathrm{A}$ units. For structure 9 instead, with methyl groups attached to the D unit and fluorine atoms in the A unit, functionalization doesn't seem to have any appreciable effect, at least in the band gap, which is essentially the same as structure 1 with both hydrogens as $\mathrm{R}_{1}$ and $\mathrm{R}_{2}$, although both HOMO and LUMO energies are shifted towards lower values. The opposite behaviour was observed for HOMO and LUMO levels of structure 6, since both of them increased after functionalization, although the effect was more intense for the HOMO (from $-4.13 \mathrm{eV}$ to $-3.75 \mathrm{eV}$ ) rather than for the LUMO (from $-3.22 \mathrm{eV}$ to $-2.98 \mathrm{eV}$ ); this is the reason why structure 6 presents the lowest band gap in the series. Structures 5 and 7 , both with bulky substituents, present almost the same band gap as structure 4 , despite the HOMO and LUMO energies being different by $\sim 0.3 \mathrm{eV}$ : this effect is probably due to the steric hindrance caused by the t-butyl and t-butoxy groups that leads to slightly non-planar structures, and therefore a lower degree of conjugation and higher HOMO and LUMO energies. Fluorination on thiophene does seem to have an effect (in contrast to what can be observed for repeat units), causing an increase in the band gap of $0.12 \mathrm{eV}$ and an appreciable decrease in both HOMO and LUMO levels, when comparing structure 2 with 11. The replacement of BTZ with NTZ also has an effect, in particular on the HOMO and LUMO energies: when comparing structures 2 and 12 (which have the same functional groups), it is clear how naphthothiadiazole shifts both the frontier orbitals towards lower values, while the band gap doesn't seem to be strongly affected. Structure 14, formed by an alternating fluorinated benzothiadiazole and a single thiophene unit, presents almost the same band gap as structure 23 (PffBT4T-2OD), that consists of the same fluorinated benzothiadiazole unit, but alternated with four thiophene units, despite both HOMO and LUMO energies being higher in the latter. Of great importance is also the HOMO energy offset between the 


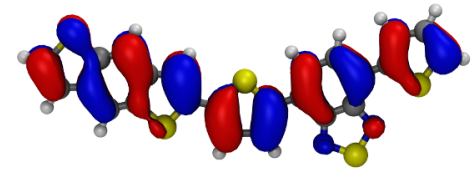

(a) HOMO of structure 1

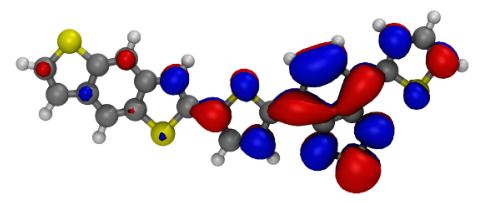

(d) LUMO of structure 1

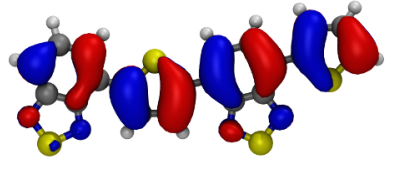

(b) HOMO of structure 13

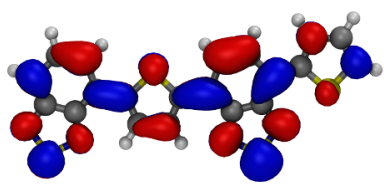

(e) LUMO of structure 13

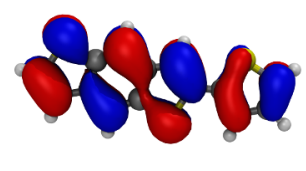

(c) HOMO of structure 17

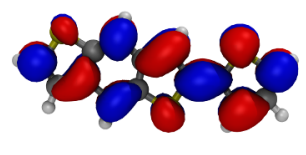

(f) LUMO of structure 17

Figure 5: Isovalue surface plots of HOMO and LUMO of repeat units 1, 13, and 17.

donor and the acceptor blocks: as previously mentioned, a well-performing material must have non-vanishing hole mobility, and therefore valence bands of acceptor and donor blocks should be similar. Structure $13(\mathrm{R}=\mathrm{H})$ for example matches quite well with structures 17 $(\mathrm{R}=\mathrm{H})$ and $20\left(\mathrm{R}=\mathrm{CH}_{3}\right)$, structure 14 and 18 , both with $\mathrm{R}=\mathrm{F}$, have almost exactly the same HOMO energy, and structure $15\left(\mathrm{R}=\mathrm{OCH}_{3}\right)$ matches reasonably well with structure $20\left(\mathrm{R}=\mathrm{CH}_{3}\right)$. Structure $16(\mathrm{R}=\mathrm{CN})$ presents a really deep HOMO level and it doesn't seem to match with any of the other blocks, perhaps only with structure 21, which has the same $\mathrm{CN}$ functional group, although the difference in energy is around $0.30 \mathrm{eV}$.

The localization of frontier orbitals in both repeat units and polymers was also investigated, as shown in Figure 5: the isosurface plots of HOMO and LUMO for structures 1-12 show a general pattern where HOMO is mostly delocalized throughout the repeat units (polymers) backbone, while LUMO is mostly localized on the acceptor units (i.e. BTZ and NTZ), and this is typical for most of the D-A polymers present in literature. ${ }^{31,32}$ Some functional groups, however, are able to significantly influence the orbitals' localization, and this is the case of structures 6 and 8. Structure 6 presents a localized HOMO on the BDT donor unit due to the electron-donating $\mathrm{OCH}_{3}$ group attached to it, while in structure 8 , that differs from the previous one only for the CN group attached to the D unit, both HOMO and LUMO are delocalized along the chain; this may suggest that for structure 8 an intramolecular charge separation is less likely to be observed, if observed at all. Structures 
13-16, composed of alternated benzothiadiazole and thiophene moieties, all show delocalized HOMO and LUMO, regardless of the functional group. This behavior is not typical for D-A copolymers (in this case, thiophene rings can be regarded as donor groups), as for these structures frontier orbitals are as delocalized as in homopolymers. The same localization can be observed for structures 17-21 composed of benzodithiophene and thiophene.
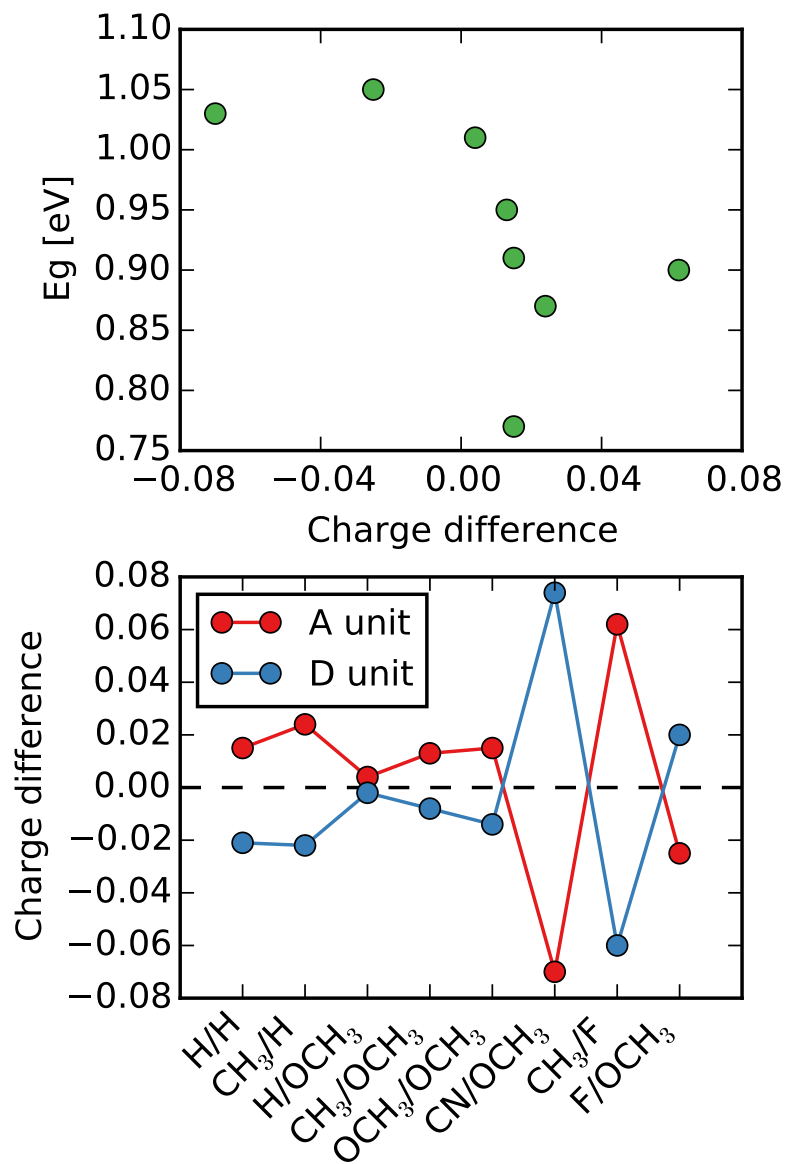

Figure 6: Electron redistribution as a function of the chemical substituents (bottom graph), and band gap of copolymers as a function of the electron redistribution (top graph). The electron redistribution was defined so that positive values correspond to an increase in the number of electrons, while negative values correspond to a decrease. The names of the polymers are in the $\mathrm{D} / \mathrm{A}$ format, i.e. the first functional group is the one attached to the $\mathrm{D}$ unit, while the second is the one attached to the $\mathrm{A}$ unit.

Also, Mulliken population analysis was performed in order to investigate if and how charge redistribution between $\mathrm{D}$ and A blocks may correlate with the band gap trend previously shown and discussed. Charges were computed on a set of polymers consisting of 8 different D- 
A structures and the corresponding D- and A-only structures; the charge difference between A and D blocks when bonded together and when bonded with themselves was computed in order to provide an estimate of how electrons redistribute after copolymerization. The charge redistribution was then defined as the sum of the charge difference of D and A blocks separately (note that the total sum of the charge difference between D and A blocks is zero, as expected). From the bottom plot of Figure 6 it can be observed that in most of the cases electrons move from the donor to the acceptor unit, even when blocks have the same functional groups (as in the $\mathrm{H} / \mathrm{H}$ and $\mathrm{OCH}_{3} / \mathrm{OCH}_{3}$ cases), showing that the BTZ-T moiety has a natural tendency of attracting electrons. However, if a strong electron withdrawing group, like $\mathrm{F}$ or $\mathrm{CN}$, is attached to the BDT-T moiety, electrons flow in the opposite direction, that is from the A to the D block. The top plot of Figure 6 shows the band gaps of the eight D-A copolymers as a function of the charge redistribution: it is evident how there doesn't appear to be any kind of correlation between these two quantities.

Experimental values of polymer band gaps, where available, were also compared with the results both obtained from simulations using PBE and B3LYP functionals. While PBE results were computed under periodic boundary conditions, B3LYP values were obtained with open boundary conditions due to the impossibility of performing periodic calculations with hybrid functionals in the current version of ONETEP, as previously mentioned in Section 2; therefore, the B3LYP results refer to oligomers consisting of 5-6 repeat units each. The number of repeat units for this set of oligomers was chosen after performing a convergence analysis of the band gap against the number of units, so that the error in the band gap of these oligomers was between $0.04-0.03 \mathrm{eV}$. Figure 7 and Table 3 show that, despite their absolute values being different due to the use of PBE and B3LYP functionals, the band gaps obtained with ONETEP follow the same trend as in experiments. As expected, the PBE provides an underestimate of the band gap, while the hybrid functional is a slight overestimate, with the only exception of structure 15 whose computed band gap is $0.01 \mathrm{eV}$ lower than the experimental one; however, this deviation is small and within the experimental 
error. These two functionals provide a lower and a (slight) upper bound respectively for the experimental band gaps, even if it is worth remembering that it is not certain that B3LYP will produce overestimates of those quantities: formally Hartree-Fock calculations would provide the upper bound due to the way that the exchange interaction is calculated; B3LYP contains only a fraction of Hartree-Fock exchange so this effect is moderated. Finally, it is also worth noting that the difference between the experimental and the theoretical values is rather constant, given the experimental error in the determination of optical band gaps.

Table 3: Comparison between numerical values (in $\mathrm{eV}$ ) of theoretical and experimental $E_{g}$ of polymers.

\begin{tabular}{cccc}
\hline polymer & exp & PBE & B3LYP \\
\hline 4 & $1.77^{14}$ & 0.95 & 1.83 \\
15 & $1.72^{33}$ & 0.87 & 1.71 \\
22 & $1.58^{a}$ & 0.77 & 1.60 \\
23 & $1.65^{b}$ & 0.83 & 1.66 \\
\hline
\end{tabular}

${ }^{a}$ See ref. $21^{b}$ See ref. 20

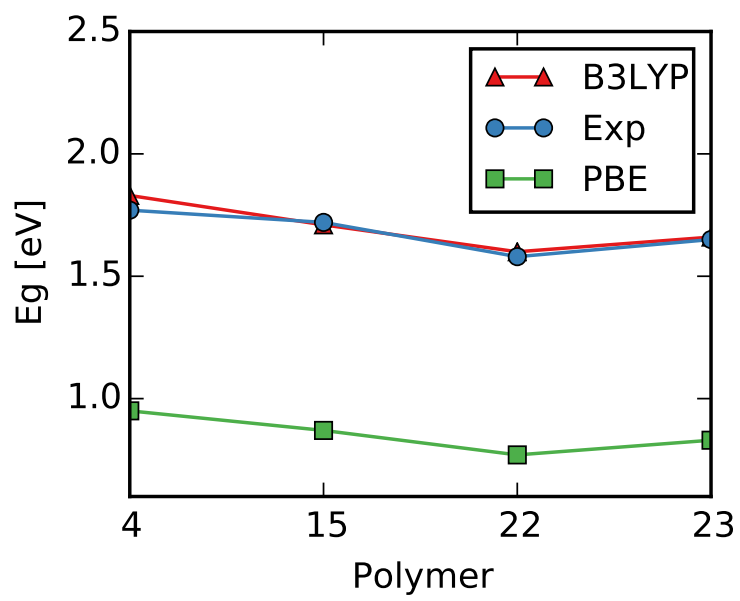

Figure 7: Comparison between theoretical and experimental band gaps of polymers.

\section{Long chain oligomers}

As previously mentioned, the five long chain oligomers have all the same functional groups in $\mathrm{R}_{1}$ and $\mathrm{R}_{2}$ positions $\left(\mathrm{CH}_{3}\right.$ and $\mathrm{OCH}_{3}$ respectively), and therefore first three oligomers taken 


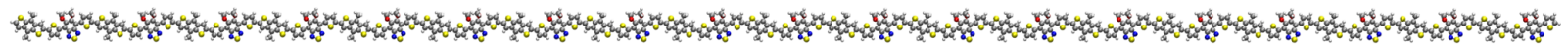

Oligomer

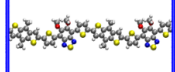

Dimer in PBC

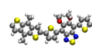

Monomer

Figure 8: Ball and stick representation of structure 4 oligomer, dimer in PBC, and repeat unit.

from structures $4\left(\mathrm{R}_{1}=\mathrm{CH}_{3}, \mathrm{R}_{2}=\mathrm{OCH}_{3}\right), 15\left(\mathrm{R}=\mathrm{OCH}_{3}\right)$ and $20\left(\mathrm{R}=\mathrm{CH}_{3}\right)$ were built, and the results obtained after geometry optimization were compared with the corresponding ones obtained in periodic boundary conditions, as shown in Table 4. The oligomers of structures 4, 15, and 20 are composed of 19, 18, and 20 repeating units respectively, and Figure 8 shows a comparison between structure 5 oligomer, a dimer in periodic boundary conditions, and finally a single repeat unit. The HOMO and LUMO energies of the oligomers were found to be in very good agreement with the ones obtained in PBC; this is further confirmation that these polymers have very flat bands: for structures 1-11 and 17-21 only one repeating unit in the unit cell is enough to obtain reasonably converged energies, while for structures 13-16 at least two units are necessary.

Figure 9 shows a comparison between density of states (DOS) centered around the energy gaps of single repeat units, polymers obtained in PBC using just one repeating unit, polymers in PBC but with two repeating units, and finally oligomers with 19, 18, and 20 units. For each of the structure it was observed that the DOS between repeat units and oligomers are significantly different: not only the HOMO and LUMO energies get closer because of the effect of conjugation, leading to a narrowing in the energy gaps, but a broadening of the states can also be observed, especially for structures 15 and 20. This is consistent with the fact that while for single repeat units the energy levels are well defined and separated, for oligomers they become significantly closer to one another, and eventually a semiconductor 

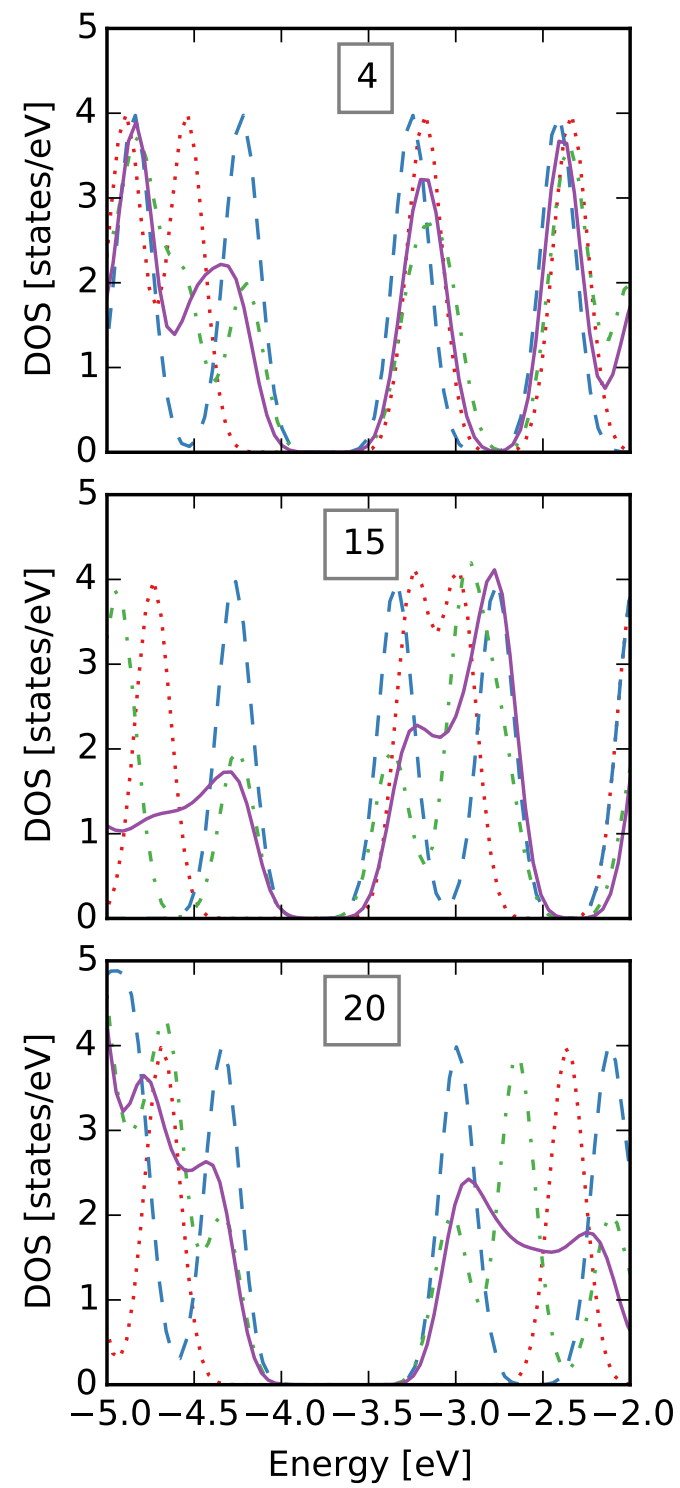

Figure 9: Comparison between density of states of repeat units (dotted red line), polymers obtained in $\mathrm{PBC}$ with 1 single unit in the repeating cell (dashed blue line), polymers obtained in PBC with 2 units (dotted and dashed green line), and oligomers (solid purple line) of structures 4 (top), 15 (middle), and 20 (bottom) respectively. 
Table 4: Energies of oligomers obtained after geometry optimization, compared with the ones obtained with periodic boundary conditions.

\begin{tabular}{lccc}
\hline & HOMO $(\mathrm{eV})$ & LUMO $(\mathrm{eV})$ & $E_{g}(\mathrm{eV})$ \\
\hline \multicolumn{3}{c}{ structure 4} \\
\hline PBC (1 unit) & -4.20 & -3.25 & 0.95 \\
PBC (2 units) & -4.20 & -3.26 & 0.94 \\
Oligomer & -4.20 & -3.28 & 0.92 \\
\hline \multicolumn{3}{c}{ structure 15} \\
\hline PBC (1 unit) & -4.28 & -3.34 & 0.94 \\
PBC (2 units) & -4.24 & -3.38 & 0.87 \\
Oligomer & -4.20 & -3.34 & 0.86 \\
\hline & \multicolumn{3}{c}{ structure 20 } \\
\hline PBC (1 unit) & -4.34 & -3.01 & 1.33 \\
PBC (2 units) & -4.33 & -3.02 & 1.31 \\
Oligomer & -4.32 & -3.03 & 1.29 \\
\hline
\end{tabular}

band structure is observed. When comparing DOS of oligomers and polymers obtained in PBC it's also clear that in general a repeating cell with just one unit (or two units, in the case of structure 15) is enough to obtain converged numerical values of the HOMO and LUMO energies, even if the density of states overall are clearly different.

Results of the ground state energies of oligomers obtained with different reactivity ratios are shown in Table 5. Structures D10-A0.1 and D5-A0.2, composed mostly of D blocks, Table 5: Energies of oligomers obtained after geometry optimization with different reactivity ratios for $\mathrm{D}$ and $\mathrm{A}$ blocks. ${ }^{a}$

\begin{tabular}{lcccc}
\hline & no. atoms & HOMO $(\mathrm{eV})$ & LUMO $(\mathrm{eV})$ & $E_{g}(\mathrm{eV})$ \\
\hline Pure D & 1046 & -4.32 & -3.03 & 1.29 \\
Pure A & 1042 & -4.20 & -3.34 & 0.86 \\
Alternating D-A & 1047 & -4.20 & -3.28 & 0.92 \\
\hline D10-A0.1 & 1156 & -4.29 & -3.29 & 1.00 \\
D5-A0.2 & 1150 & -4.28 & -3.26 & 1.02 \\
D0.2-A5 & 1069 & -4.23 & -3.36 & 0.87 \\
D0.1-A10 & 1054 & -4.22 & -3.36 & 0.87 \\
D1-A1 & 1096 & -4.25 & -3.34 & 0.91 \\
\hline
\end{tabular}


present HOMO energies close to the one of the Pure D oligomer, while LUMO energies are closer to the one of the Pure A structure; the band gaps, with values of $1.00 \mathrm{eV}$ and $1.02 \mathrm{eV}$ respectively, present intermediate values between the ones of Pure D and Pure A structures, and they are both wider than the one of the alternating D-A oligomer. Structures D0.1-A10 and D0.2-A5, that consist mostly of A blocks, on the other hand present both HOMO and LUMO energies that are closer to the one obtained for the BTZ-T oligomer; both energy gaps in turn present almost the same values as the one of the BTZ-T oligomer, and therefore smaller in magnitude than the one of the alternated D-A oligomer. Finally structure D1-A1 (i.e. a totally random copolymer) presents a HOMO level that is almost the average between that predicted for the Pure D and Pure A oligomers, while the LUMO energy is exactly the same as the one of the Pure A structure. Small changes were observed in the HOMO and LUMO energies, in the order of $<0.05 \mathrm{eV}$, when comparing the values between the alternated copolymer and the totally random one; the same observation was found to be true also for the energy gap, with a change of $\sim 0.01 \mathrm{eV}$. It seems therefore that the "sequence effect" 34 (i.e. the order of the blocks in the chain) does not play an important role, at least for this class of materials.

Frontier orbitals of all the oligomers studied present the same localization behavior as DA polymers: HOMO is mostly delocalized on the oligomer backbone, while LUMO is strictly localized on the acceptor groups of the chain.

\section{Conclusions}

D-A copolymers are widely used in the field of organic photovoltaics because of their versatility: HOMO and LUMO energies, as well as the band gap, can be carefully tuned in different ways in order to obtain materials with suitable and desired optical properties (such as a low band gap), with the aim of boosting the efficiency of this kind of devices. This study in particular focused on the effect of composition on a class of statistical copolymers, and the 
model structures used in this paper consisted of analogues and blocks of the high-performing PBTZT-stat-BDTT-8 developed by Merck.

The effect of composition was studied by modifying the $\mathrm{D} / \mathrm{A}$ block ratio in the polymer chain, without changing the functional groups. The block ratio was modified by setting different values of reactivity ratios and the final compositions of the oligomers were obtained by using the Mayo-Lewis equation as implemented in the Materials Studio software. This study was enabled by the linear-scaling reformulation of DFT in the ONETEP code, that allowed calculation at a far larger scale than possible before: it was then possible to study a series of long chain oligomers composed of more than a thousand atoms, allowing us to obtain of well converged energy levels, which would have been impossible otherwise (previous theoretical works on this subject made use only of small tetramers ${ }^{11}$ ). Our results show that the composition plays a very important role in the determination of the electronic properties of a D-A copolymer: when varying the $\mathrm{D} / \mathrm{A}$ block ratio, changes in the energy gap of the same material were observed and were in the order of $\sim 0.1 \mathrm{eV}$, an effect that is to some extent comparable to changing the functional groups. The band gap of the studied material ranged between $\sim 1 \mathrm{eV}$ (in the case where more $\mathrm{D}$ units were present in the chain) and $0.87 \mathrm{eV}$ (in the opposite case, where a higher number of A units was present in the chain). From these results it is clear that modifying the block ratio of the polymer (in other words, modifying the synthesis strategy) leads to structures with significantly different electronic properties, without the need to change the functional groups. In addition, at least for this class of statistical copolymers, the sequence effect doesn't seem to significantly affect the HOMO, LUMO and band gap, since only a small change in these values was observed when comparing the energies of an alternating D-A oligomer with a completely random one, built by assigning the same reactivity ratios for both blocks.

An extensive study on the electronic properties of this class of materials was also performed: in order to understand how the electronic structure of PBTZT-stat-BDTT-8 is susceptible to functionalization, the effect of a number of substituents attached to its donor 
and acceptor units was investigated. Different functional groups were tested, both electronwithdrawing and electron-donating. In general electron-withdrawing groups were found to cause a decrease in both HOMO and LUMO energies and in both A and D units, while electron-donating groups on the contrary showed the opposite effect. However, when combining the donor and the acceptor units together it was hard to rationalize the effect of specific substituents in terms of HOMO and LUMO energies: this suggests that it is unlikely to know a priori using chemical intuition how a functional group can influence the energy levels of a D-A copolymer, and therefore computational modelling is a useful and essential tool. This finding is also supported by the fact that no correlation could be found between the band gaps and the charge redistribution of the materials studied. The results were also supported by comparing computed energy gaps, using both hybrid and GGA functionals, with the corresponding experimental ones present in literature, and despite the obtained theoretical values with PBE being smaller in magnitude than the experimental ones (or slightly higher and/or comparable in the case of B3LYP), we were able to accurately reproduce the experimental trend.

The results shown in this paper can be used as a starting point for the design of efficient D-A statistical copolymers with suitable electronic properties for OPV devices. However, more in-depth studies need to be done in order to address more complex problems regarding these kinds of materials, such as the effect of chain-chain stacking and the interaction between these polymers and fullerenes.

\section{Supporting Information Available}

Extrapolation of band gaps by linear and quadratic fit for a set of structures $(4,15,22$, 23), as a comparative example to results obtained with PBC and, in some cases, to results of the long chain oligomers. This material is available free of charge via the Internet at http://pubs.acs.org/. 


\section{Acknowledgement}

G.B. acknowledges the support of Merck and an EPSRC Doctoral Training Centre grant (EP/L015382/1) for his PhD studentship. H.X. thanks Lanzhou University of Technology (LUT) for a scholarship from the LUT Training Program of the National and Gansu Provincial Excellent Doctoral Dissertations. H.X. also acknowledges the support of the National Natural Science Foundation of China (grant numbers 11164014 and 11364025). J.D. would like to thank EPSRC (grant numbers EP/J015059/1 and EP/K039156/1) for his postdoctoral research funding. The calculations were carried out on the Iridis4 Supercomputer of the University of Southampton. Some calculations were also carried out at the TASK Computer Centre in Gdańsk, Poland (supported in part by the PL-Grid Infrastructure, grant POIG.02.03.00-00-096/10).

\section{References}

(1) Mazzio, K. A.; Luscombe, C. K. The Future of Organic Photovoltaics. Chem. Soc. Rev. 2015, 44, 78-90.

(2) Knapp, K.; Jester, T. Empirical Investigation of the Energy Payback Time for Photovoltaic Modules. Solar Energy 2001, 71, 165-172.

(3) Espinosa, N.; Hösel, M.; Angmo, D.; Krebs, F. C. Solar Cells With One-Day Energy Payback for the Factories of the Future. Energy Environ. Sci. 2012, 5, 5117-5132.

(4) Yu, G.; Gao, J.; Hummelen, J. C.; Wudl, F.; Heeger, A. J. Polymer Photovoltaic Cells: Enhanced Efficiencies via a Network of Internal Donor-Acceptor Heterojunctions. Science 1995, 270, 1789-1791.

(5) Lee, S.-H.; Kim, D.-H.; Kim, J.-H.; Lee, G.-S.; Park, J.-G. Effect of Metal-Reflection and Surface-Roughness Properties on Power-Conversion Efficiency for Polymer Photovoltaic Cells. J. Phys. Chem. C. 2009, 113, 21915-21920. 
(6) Lee, S.-H.; Kim, J.-H.; Shim, T.-H.; Park, J.-G. Effect of Interface Thickness on Power Conversion Efficiency of Polymer Photovoltaic Cells. Electron. Mater. Lett. 2009, 5, $47-50$.

(7) Kroon, R.; Lenes, M.; Hummelen, J. C.; Blom, P. W. M.; de Boer, B. Small Bandgap Polymers for Organic Solar Cells (Polymer Material Development in the Last 5 Years). Polymer Reviews 2008, 48, 531-582.

(8) Kularatne, R. S.; Magurudeniya, H. D.; Sista, P.; Biewer, M. C.; Stefan, M. C. DonorAcceptor Semiconducting Polymers for Organic Solar Cells. J. Polym. Sci. A Polym. Chem. 2013, 51, 743-768.

(9) Nunzi, J.-M. Organic Photovoltaic Materials and Devices. C. R. Phys. 2002, 3, 523542.

(10) Slama-Schwok, A.; Blanchard-Desce, M.; Lehn, J. M. Intramolecular Charge Transfer in Donor-Acceptor Molecules. J. Phys. Chem. 1990, 94, 3894-3902.

(11) Hung, Y.-C.; Chao, C.-Y.; Dai, C.-A.; Su, W.-F.; Lin, S.-T. Band Gap Engineering via Controlling Donor-Acceptor Compositions in Conjugated Copolymers. J. Phys. Chem. $B$ 2013, 117, 690-696.

(12) Scharber, M. C.; Mühlbacher, D.; Koppe, M.; Denk, P.; Waldauf, C.; Heeger, A. J.; Brabec, C. J. Design Rules for Donors in Bulk-Heterojunction Solar Cells-Towards 10\% Energy-Conversion Efficiency. Adv. Mater. 2006, 18, 789-794.

(13) Berny, S.; Blouin, N.; Distler, A.; Egelhaaf, H.-J.; Krompiec, M.; Lohr, A.; Lozman, O. R.; Morse, G. E.; Nanson, L.; Pron, A. et al. Solar Trees: First Large-Scale Demonstration of Fully Solution Coated, Semitransparent, Flexible Organic Photovoltaic Modules. Adv. Sci. 2016, 3, 1500342. 
(14) Menelaou, C.; Tierney, S.; Blouin, N.; Mitchell, W.; Tiwana, P.; McKerracher, I.; Jagadish, C.; Carrasco, M.; Herz, L. M. Effect of Nanocrystalline Domains in Photovoltaic Devices With Benzodithiophene-Based Donor-Acceptor Copolymers. J. Phys. Chem. C 2014, 118, 17351-17361.

(15) Duan, C.; Gao, K.; van Franeker, J. J.; Liu, F.; Wienk, M. M.; Janssen, R. A. J. Towards Practical Useful Polymers for Highly Efficient Solar Cells via a Random Copolymer Approach. J. Am. Chem. Soc. 2016, 138, 10782-10785.

(16) Skylaris, C.-K.; Haynes, P. D.; Mostofi, A. A.; Payne, M. C. Introducing ONETEP: Linear-Scaling Density Functional Simulations on Parallel Computers. J. Chem. Phys. 2005, 122, 084119.

(17) Hine, N. D. M.; Robinson, M.; Haynes, P. D.; Skylaris, C.-K.; Payne, M. C.; Mostofi, A. A. Accurate Ionic Forces and Geometry Optimization in Linear-Scaling Density-Functional Theory With Local Orbitals. Phys. Rev. B 2011, 83, 195102.

(18) Skylaris, C.-K.; Mostofi, A. A.; Haynes, P. D.; Diéguez, O.; Payne, M. C. Nonorthogonal Generalized Wannier Function Pseudopotential Plane-Wave Method. Phys. Rev. B 2002, 66, 035119.

(19) Mostofi, A. A.; Haynes, P. D.; Skylaris, C.-K.; Payne, M. C. Preconditioned Iterative Minimization for Linear-Scaling Electronic Structure Calculations. J. Chem. Phys 2003, 119, 8842 .

(20) Hu, H.; Jiang, K.; Yang, G.; Liu, J.; Li, Z.; Lin, H.; Liu, Y.; Zhao, J.; Zhang, J.; Huang, F. et al. Terthiophene-Based D-A Polymer With an Asymmetric Arrangement of Alkyl Chains That Enables Efficient Polymer Solar Cells. J. Am. Chem. Soc. 2015, 137, $14149-14157$.

(21) Kawashima, K.; Osaka, I.; Takimiya, K. Effect of Chalcogen Atom on the Properties of 
Naphthobischalcogenadiazole-Based $\pi$-Conjugated Polymers. Chem. Mater. 2015, 27, $6558-6570$.

(22) Zade, S. S.; Bendikov, M. From Oligomers to Polymer: Convergence in the HOMOLUMO Gaps of Conjugated Oligomers. Org. Lett. 2006, 8, 5243-5246.

(23) Dassault Systèmes BIOVIA, Materials Studio, version 8.0. 2016; San Diego, CA.

(24) Mayo, F. R.; Lewis, F. M. Copolymerization. I. A Basis for Comparing the Behavior of Monomers in Copolymerization; The Copolymerization of Styrene and Methyl Methacrylate. J. Am. Chem. Soc. 1944, 66, 1594-1601.

(25) Perdew, J. P.; Burke, K.; Ernzerhof, M. Generalized Gradient Approximation Made Simple. Phys. Rev. Lett. 1996, 77, 3865-3868.

(26) Perdew, J. P.; Levy, M. Physical Content of the Exact Kohn-Sham Orbital Energies: Band Gaps and Derivative Discontinuities. Phys. Rev. Lett. 1983, 51, 1884-1887.

(27) Sham, L. J.; Schlüter, M. Density-Functional Theory of the Energy Gap. Phys. Rev. Lett. 1983, 51, 1888-1891.

(28) Becke, A. D. Density-Functional Thermochemistry. III. The Role of Exact Exchange. J. Chem. Phys. 1993, 98, 5648-5652.

(29) Lee, C.; Yang, W.; Parr, R. G. Development of the Colle-Salvetti Correlation-Energy Formula Into a Functional of the Electron Density. Phys. Rev. B 1988, 37, 785-789.

(30) Dziedzic, J.; Hill, Q.; Skylaris, C.-K. Linear-Scaling Calculation of Hartree-Fock Exchange Energy With Non-Orthogonal Generalised Wannier Functions. J. Chem. Phys. 2013, 139, 214103

(31) Risko, C.; McGehee, M. D.; Brédas, J.-L. A Quantum-Chemical Perspective Into Low Optical-Gap Polymers for Highly-Efficient Organic Solar Cells. Chem. Sci. 2011, 2, $1200-1218$. 
(32) Kim, B.-G.; Ma, X.; Chen, C.; Ie, Y.; Coir, E. W.; Hashemi, H.; Aso, Y.; Green, P. F.; Kieffer, J.; Kim, J. Energy Level Modulation of HOMO, LUMO, and Band-Gap in Conjugated Polymers for Organic Photovoltaic Applications. Adv. Funct. Mater. 2013, 23, 439-445.

(33) Lee, W.; Choi, H.; Hwang, S.; Kim, J. Y.; Woo, H. Y. Efficient Conventional- and Inverted-Type Photovoltaic Cells Using a Planar Alternating Polythiophene Copolymer. Chem. Eur. J. 2012, 18, 2551-2558.

(34) Kanal, I. Y.; Owens, S. G.; Bechtel, J. S.; Hutchison, G. R. Efficient Computational Screening of Organic Polymer Photovoltaics. J. Phys. Chem. Lett. 2013, 4, 1613-1623. 


\section{Graphical TOC Entry}

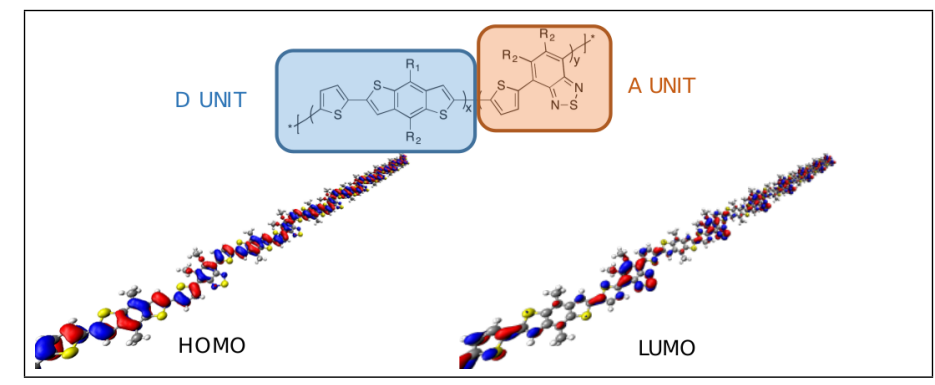

